\title{
Rhétorique et argumentation : approches croisées
}

Current Approaches to Rhetoric and Argumentation

\section{Ruth Amossy et Roselyne Koren}

\section{(2) OpenEdition}

\section{Journals}

\section{Édition électronique}

URL : https://journals.openedition.org/aad/561

DOI : $10.4000 /$ aad. 561

ISSN : 1565-8961

Éditeur

Université de Tel-Aviv

\section{Référence électronique}

Ruth Amossy et Roselyne Koren, « Rhétorique et argumentation : approches croisées », Argumentation et Analyse du Discours [En ligne], 2 | 2009, mis en ligne le 01 avril 2009, consulté le 15 février 2023.

URL : http://journals.openedition.org/aad/561 ; DOI : https://doi.org/10.4000/aad.561

Ce document a été généré automatiquement le 29 septembre 2020.

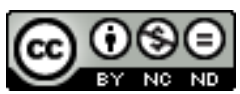

Creative Commons - Attribution - Pas d'Utilisation Commerciale - Pas de Modification 4.0 International - CC BY-NC-ND 4.0

https://creativecommons.org/licenses/by-nc-nd/4.0/ 


\title{
Rhétorique et argumentation : approches croisées
}

\author{
Current Approaches to Rhetoric and Argumentation
}

Ruth Amossy et Roselyne Koren

\section{Considérations théoriques (Ruth Amossy)}

1 L'intérêt croissant qui se manifeste aujourd'hui pour l'argumentation dans les études francophones n'est pas sans appeler quelques clarifications. Ducrot, qui a joué un rôle déterminant dans le regain des études argumentatives en France, s'y est récemment employé en différenciant nettement ce qu'il appelle «l'argumentation linguistique » et «l'argumentation rhétorique ». La première, dont il est l'un des promoteurs et le tenant, se définit contre "l'optimisme rhétorique d'Aristote et de ses innombrables successeurs » (Ducrot $2004: 32$ ) ; elle rejette l'indivision de la parole et de la raison dont témoigne le sémantisme même de logos. On sait que pour Ducrot, l'argumentation est un fait de langue et non de discours, qui intervient dans la construction du sens de l'énoncé - «Signifier, pour un énoncé, c'est orienter » (Anscombre \& Ducrot 1988) - et qui consiste en un enchaînement d'énoncés ${ }^{1}$. Cette perspective a, entre autres, fécondé l'étude des connecteurs et des topoï avant de faire place à celle des blocs sémantiques dans la théorie développée par Carel (1999). C'est cette vision de l'argumentation qu'ont essentiellement retenue les sciences du langage dans les travaux de langue française, et qui en a fait un domaine à part entière de la linguistique. Il est bien vrai cependant, comme le souligne Ducrot, que cette approche diffère profondément de "l'argumentation rhétorique " remise à l'honneur par les travaux de Perelman. En effet, Le traité de l'argumentation. La nouvelle rhétorique, publié en 1958 avec OlbrechtsTyteca, est axé sur le logos et sur une logique des valeurs. Il explore les voies par lesquelles le débat rationnel et la parole partagée peuvent substituer la recherche commune d'un accord à la violence brute.

2 La dénomination d'argumentation rhétorique, par laquelle Ducrot désigne tout ce qui relève de l'art de raisonner et de persuader par la parole, n'a cependant rien d'évident, 
et si elle permet à la pragma-sémantique de se démarquer clairement des approches discursives courantes, elle n'en pose pas moins des problèmes de fond. Le plus important tient au fait d'associer, sans problématisation aucune, argumentation et rhétorique. En effet, elles sont souvent considérées comme deux domaines d'étude différents, et ce n'est pas le moindre effet de l'œuvre de Perelman que d'avoir réuni des disciplines qui avaient été, historiquement et institutionnellement, disjointes. En d'autres termes, un titre comme Traité de l'argumentation. La nouvelle rhétorique, est en soi un coup de force qui demande des éclaircissements.

Un bref rappel, tout d'abord. Dans l'histoire de la rhétorique telle qu'on la trouve dans les manuels, il est courant de marquer la rupture qui s'est opérée entre l'art de raisonner et de persuader, d'une part, et l'art de bien dire, d'autre part, en l'attribuant à Ramus². A partir du moment où l'inventio, ou recherche des matériaux à utiliser dans le discours, et la dispositio ou organisation de ces mêmes matériaux, ont été reversées au compte du raisonnement dialectique, à savoir de la philosophie, l'elocutio relative au style est devenue l'essentiel de la rhétorique, réduisant dès lors celle-ci à la question des figures et des tropes. C'est ce que Genette (1972) a dénommé, en une expression qui a fait fortune, la "rhétorique restreinte ", montrant comment la rhétorique s'est peu à peu rétrécie aux tropes, jusqu'à se concentrer à l'époque contemporaine sur la métaphore et la métonymie. Sans doute cette vision de la rhétorique et de son enseignement a-t-elle été remise en question par les travaux de Douay (1990) qui montre, entre autres, que l'alliance des deux branches dont on clame la dissociation a persisté de 1598 à 1885 (date à laquelle la rhétorique disparaît des programmes scolaires). Il n'en reste pas moins qu'au 20e siècle la rhétorique a été longtemps perçue (et continue parfois à l'être) comme un art de l'ornement qui relève de la stylistique et non du raisonnement à visée persuasive. Elle apparait dans cette perspective comme le domaine par excellence du figural.

4 Dans la réception francophone, cette division entre art de persuader et art de bien dire a été reconduite et réinterprétée à partir des années 1970 par l'opposition de deux « Nouvelles rhétoriques». Celle de l'école de Bruxelles fondée par Perelman suit la tradition aristotélicienne en dénommant rhétorique l'étude de l'ensemble des moyens verbaux aptes à persuader, alors que celle du Groupe $\mu$ de Liège propose une approche structurale des figures qu'elle dénomme significativement Rhétorique générale (1982 [1970]). De l'aveu même des auteurs, elle relève plutôt de « la théorie littéraire, en tant qu'elle concerne au premier chef ce qu'on a appelé la fonction poétique du langage » (1982 : 202). Ainsi la nouvelle rhétorique de Perelman, élaborée dans une perspective philosophique, se concentre sur la question de la rationalité pour montrer comment un accord sur le « raisonnable » peut s'effectuer dans un cadre communicationnel. La néorhétorique du Groupe $\mu$, dans le sillage de la linguistique et en particulier de Jakobson, se concentre sur la rhétorique "non plus comme une arme de la dialectique, mais comme le moyen de la poétique (ibid.: 12), recherchant «quels sont les procédés de langage qui caractérisent la littérature " (souligné dans le texte), laquelle est avant tout conçue comme « un usage singulier du langage » (ibid. : 14) ${ }^{3}$. On voit donc comment les enjeux de la ligne de démarcation nouvelle reprennent, mais en même temps dépassent et déplacent, la rupture de bonne mémoire entre l'inventio et l'elocutio.

5 La division entre un art du raisonnement et de la persuasion, d'une part, et une théorie des figures, d'autre part, opère une redistribution des tâches qui est en prise sur une conception globale du discours. Alors que Perelman et Olbrechts-Tyteca font appel à 
des exemples littéraires aussi bien que philosophiques, juridiques ou politiques, intégrant ainsi la littérature dans l'argumentation, le projet du Groupe $\mu$ axé sur la spécificité du langage littéraire isole au contraire celui-ci pour en faire un objet d'étude à part entière. Cette division recoupe, selon Lempereur, celle qui sépare la parole efficace comme lieu de l'identité et de l'accord implicite sur les valeurs, d'une part, et la production littéraire comme lieu de la différence chaque fois renouvelée, d'autre part: " les maîtres-concepts de la division contemporaine de la rhétorique semblent donc être "écart" pour l'une et "norme" pour l'autre" (1990: 140). Lempereur, comme d'ailleurs Klinkenberg du Groupe $\mu$, insiste, dans sa contribution au volume Figures et conflits rhétoriques (1990), sur les affinités qui unissent les deux nouvelles néorhétoriques au-delà des divergences. Il n'en reste pas moins que dans la pensée contemporaine, une division, voire une incompatibilité, semble subsister entre deux conceptions dont la première se situe dans l'horizon de l'argumentation et la seconde dans l'espace de l'elocutio et du style.

Une opposition supplémentaire provient, elle aussi, de l'héritage classique et imprègne les emplois communs, en même temps que certains travaux scientifiques. Elle dérive du sens péjoratif attribué à la rhétorique par Platon, et de sa condamnation bien connue des Sophistes à nos jours : elle serait une parole persuasive et trompeuse, au regard de laquelle tous les moyens sont bons pour arriver à ses fins. Dans cette optique, la rhétorique serait une parole vaine et fausse ou, pis encore, l'art de manipuler les esprits sans souci aucun de vérité ni d'éthique. L'argumentation serait au contraire liée à la rationalité dans un souci de délibération commune. Breton reconduit clairement cette division, entrée dans l'usage de la langue, lorsqu'il écrit: "argumenter n'est pas convaincre à tout prix, ce qui suppose une rupture avec la rhétorique au sens où celleci n'est pas regardante sur les moyens de persuader » (1996:16). D'un côté, donc, un type de communication qui permet au logos de gouverner la vie de la cité; de l'autre côté, une communication en quête de pouvoir et vouée à l'efficacité pure, qui s'emploie à prendre les auditeurs au piège des mots.

7 Cette division se retrouve sous des formes plus complexes dans diverses théories de l'argumentation contemporaines qui, sans verser dans l'accusation de manipulation, opposent cependant souci de vérité et souci d'efficacité - nous y reviendrons. Elle est au contraire rejetée par les héritiers de la grande tradition aristotélicienne qui s'obstinent à maintenir une synonymie entre rhétorique et argumentation. Ainsi la nouvelle rhétorique de Perelman et Olbrechts-Tyteca se définit comme « les techniques discursives permettant de provoquer ou d'accroître l'adhésion des esprits aux thèses qu'on présente à leur assentiment» $(1970: 5)$, et Reboul voit dans la rhétorique «l'art de persuader par le discours » (1991:3). A la dichotomie entre manipulation et quête de la vérité, Perelman substitue celle de la démonstration et de l'argumentation, ellemême confondue avec la rhétorique. Selon lui, il faut distinguer entre la démonstration, qui doit arriver à la vérité par un processus logique indépendamment de tout contexte de communication, et l'usage de la parole argumentative qui utilise la langue naturelle, se situe dans un cadre communicationnel et s'emploie, dans les affaires humaines où il n'est pas de vérité absolue, à établir un accord des esprits sur ce qui peut paraitre acceptable et « raisonnable». On reprend ainsi, à nouveaux frais, le point de vue d'Aristote qui voyait dans la rhétorique le domaine du vraisemblable plutôt que de la vérité. Surtout, on arrache la rhétorique argumentative au reproche de manipulation en la présentant, non comme une simple technique destinée à faire triompher une cause, mais comme un moyen de réguler la vie sociale: elle fournit 
l'espace dans lequel la délibération et la recherche commune d'une solution plausible devient possible dans et par l'usage de la parole.

8 Ceci nous conduit à une autre opposition, qui sous-tend la différence établie entre argumentation et rhétorique dans les études anglo-saxonnes. Dans cette perspective, l'argumentation serait liée à l'étude et à l'évaluation des modes de raisonnement; elle s'attacherait aux procédures qui garantissent la validité de ces raisonnements en fonction de certaines normes de rationalité. Ainsi conçue, elle incorpore en fait l'héritage de la dialectique aristotélicienne en insistant sur la centralité du raisonnement et/ou du dialogue. La rhétorique serait, quant à elle, l'étude des échanges argumentatifs en contexte ; elle implique une prise en compte du cadre social, culturel et institutionnel de l'échange comprenant l'auditoire, la doxa de l'époque, les circonstances, etc. Pour elle, il n'est d'échange argumentatif que situé. L'argumentation se focaliserait sur le général et l'abstrait, la rhétorique sur le particulier et le concret. L'une donnerait la prééminence au logos, l'autre prendrait pleinement en compte la trilogie aristotélicienne logos (discours et raison), ethos (image de soi que l'orateur construit dans son discours), pathos (les sentiments suscités dans l'auditoire). Enfin, on pose souvent que l'une est essentiellement normative (elle élabore des critères de validité), alors que l'autre est descriptive (elle donne à voir la façon dont l'argumentation se déroule effectivement sur le terrain).

9 Le sujet a fait couler beaucoup d'encre ; il est au cœur de certains ouvrages collectifs comme les actes du colloque de OSSA (Ontario Society for the Study of Argumentation) de 1998, intitulés Argumentation \& Rhetoric, ou comme Dialectic and Rhetoric. The Warp and Woof of Argumentation, édité par van Eemeren et Houtlosser en 2002. Dans le foisonnement d'écrits auquel a donné lieu une question sans cesse reprise et toujours controversée, on exemplifiera rapidement les positions des théories de l'argumentation à partir de deux courants dominants auxquels deux des auteurs de ce numéro, Tindale et Gilbert (à l'instar de bien d'autres chercheurs) se réfèrent avec prédilection. Il s'agit de la « logique informelle » et de la " pragma-dialectique ».

10 La logique informelle est généralement conçue comme une branche de la philosophie ${ }^{4}$. Si elle se propose d'étudier les normes du raisonnement valide, elle s'écarte néanmoins de la logique formelle pour se focaliser sur les discours de la vie ordinaire : elle part de la pratique de l'argumentation en langue naturelle telle qu'elle se développe sur la place publique, dans la presse, dans les essais, etc. Johnson et Blair définissent la logique informelle comme une branche de la logique qui se propose de développer des standards non-formels, des critères et des procédures pour l'analyse, l'interprétation et l'évaluation de l'argumentation quotidienne - spontanée ou stylisée (Johnson 1998 [CDROM] : § 2.2). En d'autres termes, il s'agit d'offrir des instruments qui puissent être utilisés dans l'analyse critique des discours qui circulent sur la place publique. En plus de dégager la structure de l'argumentation dans un texte, la recherche porte sur la nature de l'argument, les types d'arguments et les critères qui permettent de les évaluer. Elle se penche conséquemment sur les fallacies ou arguments fallacieux (qu'on traduit en français par paralogismes, mais qu'on désigne aussi aujourd'hui par fallacies), c'est-à-dire sur les arguments qui paraissent valides mais en réalité ne le sont pas (Hamblin 1970). La critique des arguments fallacieux est à ce jour l'un des domaines les plus prolixes de la logique informelle, dont elle souligne les visées critiques et normatives, même lorsqu'elle assouplit considérablement les critères purement rationnels de la validité - comme le fait Walton en justifiant le bien-fondé de différents 
arguments fondés sur le sentiment (1992), dont l'appel à la miséricorde (1997) ou à la peur (2000).

11 La logique informelle, qui restreint la rhétorique à l'étude de la communication efficace, n'en essaye pas moins de jeter un pont entre les deux disciplines. Ainsi les analyses de certaines fallacies, comme les arguments ad hominem tels que les étudie Walton (1987), ne posent plus des critères absolus de validité logique, mais font dépendre la légitimité de l'argument des circonstances particulières dans lesquelles il est utilisé. La norme de rationalité et le critère de validité deviennent indissociables du contexte dans lequel l'argument est mobilisé. La contextualisation permet aussi de prendre en compte l'auditoire : Blair et Johnson parlent ainsi d'une communauté idéale d'interlocuteurs qui n'est pas sans rapport avec l'auditoire universel de Perelman (Blair \& Johnson 1987 ; Johnson 1998 : n. 8). Il n'en ressort pas pour autant qu'argumentation et rhétorique se confondent aux yeux des tenants de la logique informelle, loin de là. Johnson prend soin de définir différents critères qui permettent de les distinguer nettement. Selon lui, non seulement l'objectif de l'une est la persuasion efficace, alors que le telos de l'autre est la persuasion rationnelle ${ }^{5}$ et, qui plus est, la rationalité manifeste; mais encore la rhétorique se contente de l'argument qui porte, alors que l'argumentation se doit par principe de répondre à toute objection possible, même dérangeante ${ }^{6}$; et elle exige que les prémisses répondent au critère de vérité et ne se contentent pas, comme la rhétorique, d'acceptabilité.

12 La seconde théorie de l'argumentation, en l'occurrence la pragma-dialectique, définit également celle-ci comme une activité de la raison. Pour ce courant développé par l'école d'Amsterdam sous la houlette de Frans van Eemeren, l'argumentation est une activité verbale et sociale de la raison visant à accroître (ou à diminuer) aux yeux de l'auditeur ou du lecteur l'acceptabilité d'une position controversée en présentant une constellation de propositions destinées à justifier (ou réfuter) cette position devant un juge rationnel (van Eemeren et al. $1984: 53$, je traduis).

13 Cette approche, qui a été exposée en langue française par les auteurs dans L'argumentation aujourd'hui (Doury \& Moirand 2004), se concentre sur le processus dialectique qui permet la résolution d'un conflit d'opinion par les voies de la raison, et propose pour ce faire un modèle de la discussion critique au cours de laquelle les deux parties doivent arriver à un accord sur l'acceptabilité des positions débattues. Elle discerne quatre stades obligés du procès, la confrontation où le dissensus s'affirme, l'ouverture où les interlocuteurs s'engagent à défendre leurs vues après avoir vérifié qu'un terrain commun suffisant autorise l'échange, l'argumentation à proprement parler où les points de vue sont défendus, justifiés et développés, et la conclusion où les parties déterminent dans quel mesure un point de vue a été défendu avec succès. Par ailleurs, la pragma-dialectique élabore les règles de la discussion critique que les participants doivent respecter pour ne pas commettre de fallacies - normes qui assurent moins la bonne marche du raisonnement logique en soi, que celle de l'échange visant à la résolution des conflits. Ainsi, par exemple, chaque partie doit laisser l'autre exprimer son point de vue, ou ses doutes sur un point de vue; chacune doit défendre le sien si l'autre le lui demande; toute attaque doit concerner quelque chose qui a été effectivement avancé par l'autre, etc. Il y a là « dix commandements » qui permettent seuls de surmonter les divergences par les voies du dialogue raisonné.

14 Si l'approche est expressément rationaliste et normative, elle n'en tente pas moins, à son tour, d'intégrer une composante rhétorique dans son cadre propre. Van Eemeren et 
Houtlosser déplorent en effet qu'un "gouffre » continue à séparer les tenants d'une conception dialectique, d'une part, et les rhétoriciens, d'autre part (ibid. : 53). Pour pallier à ce manque, ils proposent depuis quelques années la notion de «strategic manoeuvering " ou "ajustement stratégique ", qui permet à l'argumentateur de mettre en œuvre des procédés contribuant à faire triompher sa cause. C'est à ce stade qu'intervient la composante rhétorique qui, selon eux, n'est pas nécessairement incompatible avec le déroulement de l'argumentation rationnelle. En effet, rien n'empêche le désir d'efficacité de s'harmoniser avec la recherche d'une résolution valide. Dans certains cas, néanmoins, il peut y avoir tension, et il importe alors de distinguer les procédés valides de ceux qui ne le sont pas, c'est-à-dire de ceux qui constituent un " dérapage » dans la mesure où ils dérogent aux règles auxquelles doit se soumettre chaque étape de la discussion critique. C'est dans cette optique que la pragma-dialectique réconcilie la théorie de l'argumentation envisagée comme une démarche permettant de résoudre les différends par la raison, et la rhétorique conçue comme recherche de l'efficacité au service d'une cause. La seconde, on le voit, est ici entièrement subordonnée à la première. Elle s'insère dans son cadre pour y apporter sa contribution: elle peut en effet rendre compte de phénomènes interactionnels qui échappent aux règles de l'échange raisonné, autorisant ainsi une analyse plus fine de l'échange argumentatif. Cette approche est bien exemplifiée dans ce numéro par l'article de Francisca Snoek Henkemans qui étudie la prétérition comme moyen particulier d'ajustement stratégique dans le cadre d'une discussion critique.

La rhétorique, qui délaisse les modèles idéaux au profit des échanges réels en contexte et ne fixe pas de normes de validité absolues, est-elle pour autant vouée à la contingence des cas particuliers, voire réduite au seul souci d'efficacité pratique ? C'est ce que nient bien des chercheurs qui y voient, au contraire, l'espace privilégié où se négocient les différences entre les humains: elle est, selon eux, le terrain par excellence des interactions qui construisent les identités et régulent le corps social. Dans cette perspective, l'importance de l'argumentation réside moins dans ses procédures de validité logique, que dans sa capacité à intervenir dans les affaires publiques et dans la vie réelle. C'est la façon dont elle investit le discours en situation qui prime. Plutôt que d'être isolées dans une autonomie factice, les procédures rationnelles doivent donc être étudiées dans le cadre de l'échange verbal dans toutes ses dimensions - logiques mais aussi institutionnelles, culturelles ou affectives. Qui plus est, le discours argumentatif doit être analysé tel qu'il se déroule effectivement dans des cas précis. Ce point de vue ne voit dans l'étude des arguments et des processus du raisonnement que l'une des parties du projet rhétorique dans son ensemble. Dans une inversion radicale des hiérarchies, ce sont alors les théories de l'argumentation qui sont subordonnées à la rhétorique.

Telle est la position adoptée, dans l'ensemble de leur travail, par deux des auteurs de ce numéro, Leff et Tindale. Dans son excellent ouvrage Rhetorical Argumentation (2004), ce dernier insiste sur le caractère par définition adressé et dialogique de l'argumentation. Définissant la «situation argumentative» comme une triade composée de l'argumentateur, de l'auditoire et de l'argument, il pose que l'argument est en grande partie déterminé par l'interrelation des deux premières instances, et de ce fait situé dans une perspective communicationnelle. C'est ce qui explique, à ses yeux, pourquoi la rhétorique est la base et la condition des aspects logique et dialectique de l'argumentation qui étudient, quant à eux, l'argument hors contexte. Nourri de logique informelle, Tindale ne rejette pas la question des normes d'évaluation; mais il prend 
soin de les fonder sur des critères d'acceptabilité plutôt que de vérité. Choisissant de façon plus radicale le camp de la rhétorique, Leff considère qu'analyser les arguments comme produits isolés permet sans doute de «rendre compte de l'argumentation de façon complexe, technique et précise", mais ne contribue guère à comprendre son fonctionnement dans la pratique. Pour lui, l'argument rationnel ne fait sens que dans sa relation aux controverses et désaccords qui participent de l'expérience du monde réel, lesquels ne peuvent à leur tour être dissociés des situations dans lesquelles ils émergent et se développent (2002: 61).

17 Ces approches inspirées de Perelman, mais aussi de la rhétorique classique ou de Bakhtine, se rapprochent de certains travaux francophones qui se sont développés en parallèle, généralement dans une ignorance mutuelle des acquis respectifs. Il s'agit, dans le cadre des sciences du langage contemporaines, des différentes recherches effectuées dans une perspective purement descriptive qui se fonde sur l'analyse de corpus oraux et écrits. L'objectif y est d'explorer le fonctionnement de l'argumentation non pas dans la langue, comme chez Ducrot, mais dans le discours, qu'il s'agisse de discours monologaux - allocution télévisée, essai, article scientifique, etc. - ou d'interactions effectives - conversation quotidienne, débat télévisé, etc. Ainsi Plantin, qui propose un modèle dialogal de l'argumentation fondé sur la « mise en contradiction active des discours autour d'une question» (2005: 34), propose comme horizon de recherche de "véritables études de cas, nécessitant le recueil de vastes corpus mixtes, oraux et écrits" (ibid.: 55). Il a lui-même travaillé sur des corpus de conversations authentiques, mobilisant les principes de l'analyse interactionnelle développés à cet effet pour explorer l'argumentation dans les échanges oraux de la vie quotidienne. Amossy, qui définit au contraire l'argumentation comme la capacité globale du verbe à infléchir, modifier ou renforcer des façons de voir et de comprendre le monde, considère quant à elle l'argumentativité comme un trait inhérent au discours et propose de l'étudier dans le cadre de l'analyse du discours «à la française » (2006 [2000]). Au-delà des différences qui les séparent, ces deux approches, comme d'ailleurs d'autres travaux d'inspiration linguistique (Adam et Bonhomme 1997; Charaudeau 2008 ; Doury 1997), proposent une analyse fine de l'ensemble d'un discours, ou d'un genre de discours, dans son épaisseur et sa complexité. C'est l'aspect discursif de l'argumentation comme échange et comme parole située qui est principalement mis en lumière dans des études sur le terrain. C'est dans ce sens que ces approches francophones croisent la critique rhétorique américaine qui, contrairement aux théories de l'argumentation présentées plus haut, se penche sur l'analyse de textes en situation et non sur des arguments décontextualisés.

Si l'argumentation rhétorique telle qu'elle apparaît aujourd'hui dans les études en langue française mobilise plus massivement les outils de la linguistique, c'est qu'elle s'inscrit souvent dans les sciences du langage, ou y reste étroitement affiliée. Il ne faut pas oublier que, contrairement aux pays anglo-saxons, et en particulier aux Etats-Unis ou au Canada, où on trouve un enseignement officiel de la rhétorique et/ou des théories de l'argumentation dans des départements divers - philosophie, speech communication, anglais - les pays francophones ne réservent aucun cursus à part entière à la rhétorique. Sans doute y trouve-t-on des recherches sur l'histoire de la rhétorique, mais elles ne se traduisent pas par un enseignement autonome. Les travaux mentionnés ici sur l'argumentation rhétorique ne se relient d'ailleurs pas à cette tradition. Si l'absence d'un enseignement disciplinaire à part entière est regrettable, il faut cependant reconnaître l'originalité des études sur l'argumentation qui découle de cette 
situation dans le domaine français. Elle se marque essentiellement dans la capacité à explorer le discours dans sa matérialité linguistique. Les tentatives d'agir sur des façons de voir et de penser sont éclairées à l'aide des instruments développés par les différents courants de la linguistique du discours - de la linguistique de l'énonciation et de l'analyse du discours à l'argumentation dans la langue de Ducrot, volens nolens mobilisée à cet effet. C'est sans doute le grand intérêt de ces travaux, qui étudient et éclairent des corpus par ailleurs au centre de diverses disciplines, en apportant leur contribution aux études de communication, aux études littéraires, aux sciences politiques et à l'histoire.

Dans l'ensemble, on peut donc voir que les positions sur les relations entre rhétorique et argumentation vont de la revendication d'une coupure radicale, à un équilibre variable entre ce qui est considéré comme deux disciplines distinctes et néanmoins complémentaires, et jusqu'à la proposition d'une fusion au sein d'une même activité verbale. Chacune de ces positions repose sur une conception différente de l'argumentation, et sur des présupposés distincts sinon divergents. D'un côté, on trouve l'argumentation dans la langue qui rejette l'argumentation rhétorique fondée sur le logos et qui se donne comme une sémantique; et, à son antipode mais campant sur le même refus de la rhétorique, la logique informelle dans ses premiers stades, vouée à l'examen du raisonnement verbal et de la validité des arguments, ou la pragmadialectique à ses débuts, étudiant les stades de la discussion critique fondée sur la raison. Par rapport à cette insistance sur la rationalité pure, ces deux mêmes courants adoptent aujourd'hui une attitude moins rigide, et tentent de réintégrer sous des formes diverses la composante rhétorique, qu'ils subordonnent à l'argumentation. De l'autre côté, on trouve des approches qui voient dans la rhétorique le cadre fondamental dans lequel il faut comprendre et insérer l'argumentation sous peine de la couper de son utilisation en situation, et donc de la vider de sa pertinence et de son sens.

20 A l'extrémité de ce pôle, il faut insister sur les courants qui se refusent à disjoindre argumentation et rhétorique. C'est, on l'a vu, ce que propose la Nouvelle rhétorique de Perelman en reconduisant la rhétorique dans son sens aristotélicien de moyens verbaux visant à entraîner l'adhésion du public. C'est aussi ce qui ressort du livre d'Adam et Bonhomme (1997) sur le discours publicitaire quand ils entendent s'appuyer sur la rhétorique classique, enrichie des acquis de la pragmatique et de la linguistique textuelle, pour aborder « une forme particulière de discours argumentatif ». C'est, de façon plus radicale, «l'argumentation dans le discours » selon Amossy (2006 [2000]), qui considère que l'argumentativité est inhérente au discours et s'y manifeste à des degrés divers, proposant dès lors une analyse du fonctionnement discursif global dans lequel des moyens verbaux relevant du logos, de l'ethos et du pathos sont mis en œuvre afin d'agir sur un auditoire. Dans cette perspective, il n'y a pas lieu de voir dans la rhétorique et l'argumentation des disciplines séparées.

21 Toutes les dernières tendances qui œuvrent au rapprochement de la rhétorique et de l'argumentation sont caractéristiques d'un développement important, sur lequel le présent numéro entend faire la lumière. Il s'agit de se distancier aussi bien de la dénégation de toute rationalité langagière décrétée par Ducrot, que de l'insistance exclusive mise sur la raison et la construction d'arguments valides. L'essentiel est désormais de prendre en ligne de compte le cadre de communication en mettant l'accent sur le locuteur et sur l'auditoire, le contexte ou la situation de discours, en même temps que l'interdiscours et la doxa - il s'agit de tenir compte du dialogisme 
inhérent à toute utilisation du langage -, le rôle de l'ethos et du pathos dans l'échange persuasif. Il nous semble aussi important de prendre en compte la centralité du fonctionnement discursif dans ses multiples aspects - du dispositif énonciatif et du genre de discours à des moyens verbaux comme les connecteurs, les choix lexicaux, les éléments doxiques ou les figures verbales.

22 Se pencher sur ces positions divergentes et suivre ces ramifications ne signifie pas simplement assurer la suprématie d'un domaine d'études ou d'une tradition sur l'autre pour des besoins purement institutionnels. Il s'agit au contraire de répondre à des questions centrales qui concernent la force de la parole, la place qu'y occupe la rationalité, le rapport qu'elle permet de nouer à l'autre, sa capacité à endiguer la violence physique. Les différentes interprétations qui sont données de la division argumentation / rhétorique répondent, chacune à leur façon, à ces questions. Elles déterminent la place de l'autre dans le discours argumentatif; elles éclairent les possibilités d'influence mutuelle et de coopération, tout en permettant de penser la confrontation et le questionnement. Elles tranchent entre la nécessité de s'adapter à un auditoire, ou au contraire de suivre des règles valables en tout lieu. Elles assignent une place toujours changeante et controversée à la raison. Est-elle une source de validité absolue qui édicte des normes universelles, ou est-elle au contraire essentiellement liée aux circonstances et aux cadres de l'échange verbal ? Elles font en même temps place à la prise en charge de jugements de valeur, et aux prises de position ancrées dans l'émotion, en se prononçant sur ses capacités à s'allier à la raison. Elles soulèvent simultanément la question des normes et des valeurs dans le partage de la parole : fautil édicter des règles pour proposer un modèle idéal, ou au contraire essayer de comprendre comment les choses se passent dans des échanges effectifs? Maintenir un souci de vérité et de validité logique, ou considérer que la régulation s'effectue sur le terrain? Qui plus est, ces différentes approches posent, mais aussi tentent de repenser, les termes dans lesquels on peut formuler le rapport de l'efficacité à la quête de solutions rationnelles.

23 Les articles réunis dans la première partie de ce numéro présentent les dernières avancées des recherches menées par des spécialistes de l'argumentation et de la rhétorique dont les travaux constituent une somme, et dont la réputation n'est plus à faire. A la suite des positions de Michel Meyer, qui ouvrent le numéro, on a choisi de mettre l'accent sur la réflexion anglo-saxonne, encore trop méconnue en France, afin de dégager son apport et de soulever la question de voir plus précisément comment elle croise les travaux qui se poursuivent dans le domaine francophone. Cette rencontre de courants qui tendent à s'ignorer mutuellement répond à la vocation de la revue Argumentation et Analyse du Discours, qui entend faire dialoguer des traditions diverses.

24 Michel Meyer, qui poursuit ses travaux à l'Université libre de Bruxelles où a enseigné Chaim Perelman, dont il a pris la succession, vient de publier une somme intitulée Principia rhetorica. Une théorie générale de l'argumentation (2008), qui vient couronner des recherches publiées dans de nombreux ouvrages, parmi lesquels on se contentera de citer ici La rhétorique (2004) et Qu'est-ce que l'argumentation? (2005). Il introduit dès le départ une distinction essentielle, qui pourrait servir de cadre à toute discussion sur le sujet, concernant le sens qu'on accorde au terme de rhétorique. En effet, Meyer la conçoit tout d'abord comme la discipline qui englobe l'argumentation; mais aussi, dans un second temps comme une somme de procédés discursifs, qui s'opposent à celle-ci. A partir de là, Meyer clarifie l'opposition de la rhétorique prise dans son sens restreint et 
de l'argumentation, à partir du principe unificateur autour duquel s'organise l'ensemble de son œuvre philosophique : la problématologie (2008 [1988]). Celle-ci a l'avantage de montrer dans toutes ses conséquences la centralité du questionnement. Dès lors qu'une question se pose, avance Meyer, on peut l'affronter à partir de l'opposition des réponses qui y sont apportées - c'est l'argumentation - ou au contraire la masquer, l'« avaler » par l'élégance du style, en la présentant comme résolue - c'est la rhétorique. L'une partirait ainsi des questions, l'autre des réponses. Cette prise de position permet de relancer sur d'autres bases la réflexion sur les oppositions raisonnement/efficacité, quête de la vérité/manipulation, pour les repenser à travers la gestion du questionnement.

Michael Leff, l'un des grands spécialistes actuels de rhétorique aux Etats-Unis, poursuit aussi bien une réflexion sur la spécificité de la discipline $(2002,2006)$, qu'un travail riche et dense sur des éléments comme la prolepse (1999), la question de l'agent (2003), ou les lieux communs (1995). Ce n'est pas par hasard que ses écrits portent sur l'analyse de textes précis comme un discours de Roosevelt, le débat Bush/ Kerry, ou la lettre de la prison de Birmingham de Martin Luther King. Leff prône ici la nécessité de ne pas séparer l'arsenal des stratégies argumentatives des cas particuliers dans lesquels elles sont mises en œuvre. Une analyse fine d'un texte de W. E. B. DuBois qui tente de définir un programme politique pour les Afro-Américains en se démarquant de son prédécesseur, T. Washington, illustre bien cette approche analytique de la rhétorique, et l'importance qu'elle accorde à la personne. A partir de la notion d'argument ad hominem, et du traitement qu'il lui fait subir en le rattachant à l'ethos, Leff montre que la spécificité de la rhétorique réside dans ce qu'elle tient compte des personnes et de la situation, alors que la dialectique, décontextualisée, s'attache à des abstractions. Si donc la rhétorique peut avoir utilement recours à celle-ci, elle lui permet en même temps de ne pas perdre contact avec la réalité, dans laquelle seule l'argumentation trouve sa finalité. C'est dans cette optique que Leff interprète la division des tâches dans une perspective de complémentarité plutôt que d'opposition.

Un troisième article sur la question présente les positions d'un théoricien de l'argumentation dont l'œuvre marque un tournant dans les conceptions de la logique informelle, Christopher Tindale. Membre du comité éditorial de Informal Logic et coéditeur des actes du colloque Argumentation \& Rhétorique (OSSA 1998), auteur en 2004 d'un ouvrage intitulé Rhetorical Argumentation, Tindale, qui considère que le caractère fondamentalement dialogique du discours argumentatif nécessite de donner la primauté à un cadre rhétorique attentif non seulement au logos, mais aussi à l'ethos, au pathos et aux topoï, se propose ici d'examiner la question de l'auditoire complexe. A une époque où l'universalisme aristotélicien n'est plus de mise, il soulève le problème posé par la diversité de ceux auxquels s'adresse le discours argumentatif. Tindale part d'une définition de l'identité comme multiple à l'intérieur de chaque individu, pour proposer une conception de l'argumentation où chaque membre de l'auditoire doit décider de la façon dont il active telle ou telle de ses identités. Dans ce cadre, l'argumentateur doit " créer les conditions de la persuasion ", à savoir parvenir à mobiliser une partie de l'identité de l'autre pour l'inciter à lui accorder la place d'honneur. Pour Tindale, les émotions comme le raisonnement jouent un rôle dans ce processus. Cette approche pose que l'argumentation, prise dans son aspect d'invitation et de collaboration, permet d'inclure les interlocuteurs les plus différents en influant sur leur choix d'entrer dans le cadre de la délibération. Il n'y aurait pas dès lors d'êtres inaccessibles à la raison, mais seulement des individus qui se refusent à entrer dans des raisonnements 
et qu'on peut tenter de persuader à le faire. Ainsi la rationalité et la diversité se voient réconciliées dans le cadre d'une conception rhétorique de l'argumentation fondée sur la primauté de l'auditoire.

Si Michael Gilbert, Professeur à l'Université d'York et auteur de Coalescent Argumentation (1997), se situe dans le champ des théories de l'argumentation, c'est en y apportant une vision peu orthodoxe selon laquelle l'émotion, l'intuition et même la réaction viscérale jouent un rôle prépondérant. L'étude de ce type d'arguments (en place d'une évaluation de la validité logique) favoriserait, selon lui, la capacité à parvenir à un accord - à trouver des options qui permettent de regrouper et de réconcilier les positions. Dans cet article, Gilbert propose de se concentrer sur les interactions quotidiennes, qui sont pour lui le paradigme même de l'argumentation dans la mesure où les participants qui doivent réagir immédiatement l'un à l'autre prennent en compte les points de vue alternatifs et peuvent de ce fait être amenés à modifier leurs positions. Il pose la centralité des objectifs, du contexte et de l'ethos dans l'interaction argumentative quotidienne qui se déroule entre « proches » pour montrer que ces trois composantes autorisent un contrôle normatif où la problématisation et la justification explicite des prises de position «à chaud ", face à l'autre, l'emportent sur les règles abstraites. Dans l'infinie variété des échanges de tous les jours, c'est la situation qui détermine les règles du jeu. Et c'est parce que la normativité découle organiquement de ces trois éléments de base qu'elle peut être, selon Gilbert, dite « naturelle».

\section{Les figures entre rhétorique et argumentation (Roselyne Koren)}

28 L'un des bénéfices majeurs de la confrontation des approches anglo-saxonnes et francophones réside dans le surcroît de visibilité accordé à la question de l'ancrage de l'argumentation dans le discours et, par conséquent, à l'argumentativité des figures. Il devient en effet possible, voire nécessaire, de lui accorder une place centrale dès lors que la critique de l'argumentation ne se limite pas à l'analyse d'arguments abstraits décontextualisés. Cet intérêt pour les figures n'est pas nouveau : linguistes, philosophes et rhétoriciens s'interrogent sur leur nature et leurs fonctions depuis toujours. De nombreux numéros de revues scientifiques leur ont été consacrés ${ }^{7}$ et il existe, de la Rhétorique d'Aristote à la Nouvelle rhétorique de Perelman, des tournants dûment répertoriés dans l'histoire de la réflexion sur l'argumentativité des figures ${ }^{8}$. Mais les questions liées aux rapports entre logique et rhétorique sont si complexes qu'on n'en finit pas de les explorer et qu'il faut le faire, comme l'affirme Johnson (1998) à chaque étape de l'histoire humaine. Douay partage ce point de vue lorsque, dans un numéro de Langue française sur "Les figures de rhétorique : actualité, reconstruction, remploi », elle incite les chercheurs attirés par cet « objet presque mythique » à se livrer à une " reconstruction aussi objective que possible du domaine ancien, sans visée récupératrice », et au "remploi, hardi et clairvoyant, dans nos constructions, sous nos hypothèses, de certains beaux concepts anciens, qui dans ce déplacement ne manqueront pas de se trouver transformés » (1994:15).

29 C'est dans le sillage de ce type de « remploi » et de "reconstruction » que ce numéro intitulé Rhétorique et Argumentation se situe. Il ne s'agit pas d'y proposer un énième classement des figures ou une thèse inédite qui résoudrait une fois pour toutes la 
question du clivage entre figures poétiques et figures à visée argumentative, argumentation dans la langue et argumentation rhétorique. Nous ne prétendons pas davantage désigner ni analyser des transformations qui seraient valables pour l'ensemble des figures. Nous souhaitons simplement éclairer les tenants et aboutissants $\mathrm{du}$ retour en force de la thèse de la fonction persuasive des figures, retour étroitement lié aux avancées de théories linguistiques contemporaines pragmatiques et/ou énonciatives, et à celles d'une partie de la recherche rhétorique anglo-saxonne ou francophone actuelle. Sur ce point également, il existe en effet - en dépit d'un phénomène de méconnaissance réciproque déjà mentionné - des lieux de convergence qui font avancer la cause du débat sur figure et persuasion, et que nous nous efforcerons de souligner.

La dissociation figures de style/figures de rhétorique joue un rôle historique et théorique central dans le débat sur la fonction argumentative des figures. Il s'agit en fait pour les tenants de l'argumentativité figurale d'en problématiser ${ }^{9}$ la pertinence et d'insister sur le fait que le principe de la dissociation est intenable. Il est d'ailleurs intéressant de noter que Klinkenberg, représentant notoire du Groupe $\mu$, affirme que ces deux rhétoriques « ne sont pas aussi éloignées l'une de l'autre que l'on a bien voulu le dire " $(1996$ : 115). Elles partageraient des points communs comme l'importance accordée à la notion d'écart et à son envers, la notion de norme, l'ancrage de la réflexion épistémologique dans les théories du langage, et les avancées contemporaines de la sémantique. S'y ajoute le rôle crucial du contexte dans l'interprétation - la rhétorique de l'argumentation comme la rhétorique des figures encourageant à examiner le lien noué entre l'énoncé et ce qui l'entoure (Klinkenberg 1996).

C'est toutefois la notion d'« ornement $»^{10}$ que des rhétoriciens comme Angenot, Meyer, Molinié et Plantin, à la recherche de preuves attestant la nécessité de ne pas dissocier fleurs de rhétorique et figures argumentatives, souhaitent revisiter. Molinié affirme ainsi qu' " une approche unifiante et totalisante des figures, dans l'univers rhétorique, engage essentiellement la problématique de l'ornement » (1994: 102-103). Or, poursuitil, « il est très difficile de penser l'ornement. En pragmatique rhétorique, la visée du discours étant tout entière la persuasion par la séduction, l'ornement constitue un concept paradoxal », « le discours ainsi mis en jeu est forcément orné » et un discours qui recourrait à dessein à une esthétique du dépouillement ou de l'anti-ornement strictement informatif ne serait pas moins rhétorique que le précédent. Ce qui conduirait nécessairement à une mise en mots "ornée » du dit, c'est l'intention commune au discours littéraire et aux autres discours sociaux de mobiliser l'auditoire et d'en solliciter la coopération. L'« ornement» ne devrait donc pas être considéré comme une procédure de décoration appliquée a posteriori au langage ordinaire pour le poétiser, mais comme un acte de langage «consubstantiel au régime rhétorique du discours $»^{11}$. «Le problème de le penser demeure » néanmoins, affirme Molinié, car il serait tentant de soutenir que "la totalité des figures se définit comme ornement", terme ambivalent, qui prête à confusion, et dont Plantin tente ici même de lever l'ambiguïté. Il réfute en effet la « conception décorative de l'ornatus " par un argument «étymologique »: "ornement " référerait à «équipement» et donc aux pratiques discursives présidant à la mise en scène inéluctable de toute parole soucieuse de capter l'attention de l'auditoire. C'est la fonction rhétorique du discours, entendue comme mode de questionnement sur la triade « Soi/ Le monde extralinguistique/ L'Autre », qui conduit, selon Meyer, à « prendre pleinement la mesure de ce qu'il faut entendre par le mot ornement : on habille une question, on la manipule, littéralement parlant, on 
amplifie la problématicité »; " il y a aussi les emportements ou le ton modéré, qui servent à l'ornementation d'une question, pour négocier la distance avec l'interlocuteur " (2008:139-140). C'est au cœur des mises en mots où se construisent les interactions entre l'encodeur et ses interprètes, dans la trame des discours sociaux, que l'argumentativité des figures, rendue possible par la sélection d'ornementa ou « équipements » adéquats, est en gestation.

La Nouvelle Rhétorique de Perelman est considérée par la majorité des rhétoriciens comme le texte fondateur qui réactualise la thèse de l'interdépendance des figures à visée esthétique et à visée persuasive. C'est à lui qu'on devrait, comme le démontre ici même Plantin, la mise en vedette de parallélismes structurels entre figures et arguments. Reboul souligne que « les auteurs du Traité de l'argumentation [...] s'efforcent de dériver, et presque de déduire, chacune des figures connues d'un certain type d'argument »; « au lieu de voir dans la figure un "écart" d'expression extrinsèque à la pensée, on en fait un élément de la pensée, un moyen de trouver ou de prouver, même si ce qu'elle trouve ou prouve n'est jamais que vraisemblable» (1986:176). Tindale (2004: 67-69), quant à lui, affirme que l'originalité de la contribution de Perelman réside dans le refus de catégoriser les figures et dans le choix d'un angle d'attaque pragmatique qui consiste à

montrer que l'effet, ou l'un des effets, que produisent certaines figures dans la présentation des données est d'imposer ou de suggérer un choix, de renforcer l'impression de présence, ou de susciter une communion avec l'auditoire (ibid. : 172, je traduis).

L'argumentativité viendrait donc aux figures quand la persuasion de l'auditoire, l'incitation à la coopération et à l'action, dépendent de pratiques discursives qui intensifient et condensent la force illocutoire. Le langage aurait alors le pouvoir d'attirer l'attention de l'autre sur des points qui seraient sans doute passés inaperçus ou dont l'importance aurait été méconnue. Il contribuerait à désigner les lieux où la rupture dans l'ordre conventionnel du langage invite l'interprète à formuler l'hypothèse qu'il existe des réalités ou des vérités, reléguées dans l'implicite, dont la figure serait le signal. Celle-ci aurait ainsi le pouvoir de faire miroiter "what could be other than it is", affirme Tindale (1998: 6). Ce qui compte donc pour Perelman, ce n'est pas un type de figure qu'on pourrait présenter comme l'emblème de la figuralité, ni le recensement de la totalité des figures dans une somme technique, mais la tentative de répondre à la question : à quel besoin essentiel la figure répond-elle ? De quel manque est-elle symptômatique? Meyer abonde dans le sens de Tindale quand il affirme que Perelman a bien montré que

les figures, dans leur diversité, présentaient une même ambition sur le plan argumentatif, à savoir renforcer la présence, frapper l'imagination sur un point précis, pour suppléer au discours littéral, trop réaliste. L'effet argumentatif des figures est de créer de la proximité, de mettre en évidence la force vive des valeurs qui unissent l'orateur et l'auditoire, de renforcer le sentiment de communauté qui peut exister entre eux. Une bonne métaphore, par exemple, c'est une vision qui impose son point de vue en s'appuyant sur une image à laquelle on ne pense pas forcément et qui, subitement, éclaire la question $(2008: 126)$.

34 Autre condition pour qu'une réactualisation de la réflexion sur la force persuasive des figures soit possible: leur intégration dans le cadre théorique des recherches en sciences du langage qui accordent une place centrale aux zones opaques et floues du sens. Ce n'est plus la transparence du rapport référentiel du mot à la chose qui stimule la recherche, mais sa complexité et son ambiguïté foncière. Celles-ci constitueraient les 
conditions de possibilité de la figuralitée ${ }^{12}$ et les figures, une fois leur structure profonde décodée, éclaireraient réciproquement le sémantisme de la langue ${ }^{13}$. «L'ambiguïté figurale ", affirme Bonhomme, "apparaît comme une cristallisation exemplaire de ce qui semble caractériser la nature profonde du langage, en dépit de tentatives pour le normaliser : la duplicité et l'équivocité » (2001-2002 : 24). Les auteurs du numéro 101 de Langue Française 1994, Les figures de rhétorique et leur actualité en linguistique, avaient déjà insisté sur le fait que la figure était « avant tout un fait de (pragma-)sémantique, quoique rarement [...] sans incidence sur la (morpho-)syntaxe» (1994:5) - sémantique à explorer à la croisée d'une "sémantique lexicale contextuelle » et d'une "sémantique des textes " (ibid: 10). C'est dans le cadre d'une «linguistique du discours", à considérer comme « performancielle» (ibid:6) que leur actualité serait appelée à émerger et qu'il serait possible de «délimiter la partie prévisible ou prédictible des figures ». C'est aujourd'hui dans une perspective énonciative et pragmatique que Rabatel (2008) situe le dossier qu'il consacre à " Figures et point de vue $»^{14}$ et aux « figures d'énonciateurs ». Le cadre général retenu est celui d'une conception "profondément dialogique » de l'analyse du discours qui

permet de penser dans la langue et à travers la dynamique discursive les rapports des locuteurs à la langue, aux interactants, aux référents, en se positionnant par rapport aux préconstruits linguistiques et aux cadres de pensée doxiques (Rabatel $2008: 15)$.

L'insistance sur la dimension dialogique interactionnelle du langage et sur la confrontation de points de vue, mais aussi sur l'ancrage de ces points de vue dans le travail doxique et/ou autonome de la référenciation, est en consonance avec ce que Meyer considère comme le cadre fondateur de la rhétorique: un questionnement existentiel où interagissent dans la trame langagière le soi, le monde extralinguistique et l'autre.

Passons à présent aux questions centrales qui préoccupent les rhétoriciens, défenseurs de l'argumentativité des figures : la figure est-elle un auxiliaire de l'argument ou peutelle constituer un argument en soi? Existe-t-il une "logique figurale»? Quels en seraient les traits distinctifs ? Il ne s'agit pas ici de présenter une image exhaustive de l'état des lieux, mais uniquement de proposer un tableau synthétique représentatif de points de vue majeurs sur la question.

La plupart des auteurs consultés distinguent ainsi avec Bonhomme $(2005: 179,181)$ entre une version «faible » et une version «forte " de la fonction argumentative des figures. Reboul (1986: 175) avait défini la problématique des degrés d'argumentativité en ces termes : « Une figure de rhétorique peut-elle être un argument, ou du moins un élément d'argumentation?»; " en quoi les figures facilitent-elles l'argumentation?", « la figure peut-elle constituer elle-même un argument? ", «l'argument n'est-il pas luimême, peu ou prou une figure?» (ibid.: 176). Ces analyses l'avaient conduit à distinguer entre "un rapport extrinsèque " - figure/argumentation - où la figure ne remplirait que la fonction d'auxiliaire et un "rapport intrinsèque " où elle s'insérerait "ellemême dans la trame de l'argumentation " (ibid.: 184).Tindale (2004: 59-69) considère ce texte comme l'un des fondements de ses propres positions. C'est en effet à Reboul (1986) qu'il se réfère dès les premières lignes du chapitre 3, «La rhétorique comme argument»: "Nous désirons maintenant inverser la situation et demander si la rhétorique, ou tout au moins des procédés rhétoriques traditionnels comme les figures, peuvent fonctionner comme arguments » (2004:59, je traduis). Du fait de ce type de problématisation, le philosophe français auteur de travaux notoires sur la rhétorique, 
est rapproché non seulement de Perelman et Olbrechts-Tyteca, mais aussi de Fahnestock, une chercheuse américaine qui a publié en 1999 un ouvrage sur les figures de rhétorique dans le discours scientifique. En s'appuyant sur ces deux références, Tindale (2004:59) se prononce en faveur de la nécessité de dépasser la thèse de la figure auxiliaire et d'explorer les cas où les figures ne se contentent pas de faciliter l'argumentation, mais constituent de facto des arguments. Il se distancie cependant de Reboul par son refus de limiter l'argumentativité de la figure à son pouvoir de persuasion et insiste sur la nécessité de démontrer que « les perspectives changent [...] à travers le discernement, la compréhension et l'accord, tous facteurs auxquels l'argumentation rhétorique contribue » (ibid. : 66). Tindale ne se situe donc pas du côté de ceux qui voient dans les figures « des supports linguistiques dont le rôle se borne à véhiculer des arguments qui leur préexistent ou qui leur sont extérieurs » (Bonhomme 2005 : 179); il se prononce en faveur d'une «version forte » semblable à celle définie par Bonhomme en ces termes :

lorsqu'elles entrent dans une argumentation, la plupart des figures sont des procédés argumentatifs à part entière, ce en quoi elles débordent le domaine de l'élocution pour celui de l'invention. Selon cette version forte, on pourrait voir dans les figures des formes condensées d'arguments (ibid. : 181).

Mais si la figure ou plus exactement un certain nombre de "figures clés $»^{15}$ peuvent être considérées dans certains contextes comme des arguments à part entière, en quoi consiste la spécificité de la "logique figurale ${ }^{16}$ ? Comment la caractériser et quels en sont les traits distinctifs? Il est important de préciser, tout d'abord, que les réponses présentées et analysées ci-dessous ne concernent qu'un petit nombre de figures et non pas la totalité des figures de rhétorique connues et répertoriées. On y retrouve bien sûr la métaphore, la métonymie et l'ironie, mais on voit percer également de nouvelles vedettes comme le polyptote (Tindale 2004 et Meyer 2008), la répétition (Molinié 1994, Tindale 2004), la prétérition (Angenot 1982, Tindale 2004, Reboul 1986 et Snoeck Henkemans 2008 ici-même), l'oxymoron (Angenot 1982, Monte 2008) et l'antimétabole (Angenot 1982, Tindale 2004 et Rabatel 2008). Ce recensement indique d'emblée que des figures de rythme ou de consonance comme la répétition ou le polyptote, immédiatement perceptibles à la surface du discours, peuvent remplir une fonction argumentative jugée aussi valide et pertinente que les figures de pensée ou de construction. Un bref tour d'horizon des argumentaires justifiant la sélection de ces figures servira d'entrée en matière au développement sur les traits spécifiques de la « logique figurale».

Le polyptote ${ }^{17}$ aurait pour visée de pointer et d'amplifier les connections et les interactions entre divers phénomènes apparemment distincts, il "souligne", selon Meyer, la « question [qui] sépare » et « la différence d'amour » dans : « je vous aime plus que vous ne m'avez jamais aimé » (2008: 133). D'après Molinié, le caractère exemplaire de la répétition serait dû, entre autres, à la "graduation suivie » d'un «régime de fonctionnement figural » inhérent à toute mise en action du discours (1994 : 110-111). Tindale insiste, quant à lui, sur le rôle joué par l'emphase dans la mobilisation de l'attention de l'auditoire, auditoire qu'il s'agit de mener, via la répétition, des prémisses aux conclusions (2004: 68 et 73). La prétérition est présentée par Angenot (1982: 240-241) comme une figure qui articule la feinte à des apparences assertives et à " des hiérarchies dans la réfutation ». Elle inspire aussi Reboul (1986:178-179) qui souligne le fait qu'en affirmant qu'on ne dira rien, on parle néanmoins de l'essentiel de ce que l'on prétendait taire. Tindale (2004:60, 80-81) classe la figure dans la catégorie des 
arguments destinés à intensifier la coopération de l'auditoire et à lui faire partager la prise en charge d'une procédure argumentative évaluative de type polémique. Snoeck Henkemans radicalise ici-même ce point de vue, puisqu'elle va jusqu'à affirmer que la prétérition permettrait au sujet d'énonciation de rejeter sur l'interprète la responsabilité de l'évaluation axiologique. L'oxymoron démontre, affirme Angenot (1982: 245), combien la thèse de la "surenchère » figurale ornementale peut être discutable en contexte polémique. Il peut y être considéré comme "l'expression condensée [d'un] malaise ». Monte affirme, quant à elle, que

tout paradoxal qu'il soit, l'oxymore est étroitement relié au tissu discursif dans lequel il s'insère et peut donc être envisagé comme un outil de cohésion textuelle. Qu'il troue de sa provocation la trame apparemment unie du discours ou qu'il garde la trace d'un parcours textuel en une formule facilement mémorisable, l'oxymore est étroitement corrélé aux figures de locuteur/énonciateur construites par le texte et qu'il contribue en retour à modeler $(2008: 52)$.

La force persuasive de l'antimétabole ${ }^{18}$ est présentée par Angenot comme liée à l'« économie expressive » et à "l'élégant parallélisme entre le rythme syntaxique et sémantique et le mouvement de la pensée » (1982: 248). Tindale, citant Fahnestock, voit dans cette figure une "premise-gathering machine", "la simplicité de son schéma permet de le reconnaître et de le compléter facilement, et Fahnestock montre la fréquence de cette figure dans le raisonnement scientifique, de Pasteur à Newton, Faraday et Lamarck» (1999: 132, je traduis). Rabatel affirme que l'antimétabole peut constituer « un point de culmination qui conclut un réseau dense d'oppositions », mais il évoque également le cas où la figure joue le rôle de "premise-gathering machine " lorsqu'il ajoute qu'elle peut aussi constituer, dans un "contexte de dialogue", « l'amorce brillante d'une idée développée ultérieurement » (2008:35). Quant à l'ironie, exemple de prédilection ${ }^{19}$ de la plupart des chercheurs, Reboul $(1986: 180)$ la considère comme la figure « qui s'intègre avec le plus d'aisance dans l'argumentation » du fait de l'un de ses objets essentiels: la gestion de l'incompatibilité dans la trame hétérogène des discours. «Le principe logique de contradiction » ne peut nous permettre de penser et d'élucider le fait que l'on puisse être simultanément "démocrate et élitiste ", « libéral (en politique) et dirigiste (en économie) », énoncer une règle et la transgresser simultanément. Berrendonner (2001-2002 : 114-115) abonde dans ce sens lorsqu'il voit dans le positionnement ironique "un double jeu énonciatif» qui a pour fin de "provoquer des inférences" interprétatives divergentes, "dans une seule et même énonciation $»^{20}$. Ceci implique, si l'on veut « sauver » la présomption de pertinence, que l'on résolve ce dilemme en "supposant un énonciateur duplice, dont le comportement contradictoire s'explique comme un faire semblant». C'est à ce type de faire semblant et à la « (dis)simulation transparente » qu'Eggs consacre ici-même son article.

Le métadiscours sur les figures recensées ci-dessus est représentatif des réponses que les chercheurs consultés donnent à la question : en quoi consiste l'argumentativité des figures? Quels en sont les traits distinctifs ? Ce qui émerge, c'est une réponse en trois points :

1. la « saillance » de la figure qui rompt la linéarité du discours ordinaire et augmente la force illocutoire des énoncés ${ }^{21}$, saillance qui peut être obtenue, entre autres, par condensation. La métaphore argumentative résulterait ainsi de la condensation ${ }^{22} \mathrm{de}$ l'analogie par assimilation du thème au phore et relégation des lois de passage dans l'implicite. L'auditoire ne peut rester aveugle, ou en tout cas il le peut difficilement, au 
signal ou indice discursif qui l'interpelle et cherche à le mobiliser, afin de favoriser le transfert de l'adhésion aux prémisses à l'adhésion aux conclusions -

2. le questionnement qui renvoie à ce qu'Angenot appelle ci-dessus un «malaise ", car cette saillance figurale n'a pas de fin décorative sauf si l'on entend par "ornement » des pratiques et des techniques discursives qui problématisent et suscitent la réflexion. Il s'agit de faire comprendre à l'énonciataire qu'il y a une difficulté à résoudre, une rupture dans l'ordre des choses et que la charge de la résolution du dilemme incombe et à l'énonciateur, et à l'énonciataire interprète, impliqués dans le questionnement à des degrés variables et divers -

3. une rhétorique de la feinte intentionnelle, du « masque » (Douay $1990: 88-89$ ) et de la «(dis)simulation transparente » qui peut avoir pour enjeu la neutralisation a priori des velléités de réfutation de l'auditoire auquel on demande de s'aligner sans discuter ${ }^{23}$, ou l'auto-protection de l'énonciateur que ses positions non-conformistes risquent d'exposer aux foudres des défenseurs de la doxa. On retrouve en l'occurrence l'un des sens étymologiques d'ornement, soulignés par Plantin ici-même - " armure ».

Bonhomme invite ses lecteurs, au moment de conclure,à envisager ce que serait un discours sans figures, qui se contenterait d'être purement "dénotatif». "On verrait alors ", affirme-t-il, "la perte irréparable que cela impliquerait pour communiquer toutes les nuances de la pensée » (2005: 261). Nous aimerions prendre le relais en affirmant que cette "perte irréparable» s'étendrait à la possibilité d'une "pragmatique de la séduction» (Molinié 1994: 102-103), à la communication interactionnelle qui a un questionnement existentiel ou la persuasion pour enjeu, mais aussi à la possibilité de «s'adresser à l'homme total, à l'homme qui pense, qui agit et qui sent » (Reboul 1991: 115). Dernière perte irréparable et certes pas des moindres : la fonction d'« armure » que remplit la figure. Si la codification des figures est nécessaire à l'intercompréhension de l'énonciateur et de son interprète, la part variable et plastique de la figure, son aptitude à l'adaptation contextuelle, au masquage et à la feinte permettent, par exemple dans le cas de l'ironie, à l'énonciateur contestataire de revêtir le masque de la "naïveté » (Berrendonner 2001-2) afin de se protéger des assauts d'un auditoire intolérant et hostile. La métaphore, quant à elle, peut constituer un coup de force dans la mesure où elle relègue des lois de passage dans le non-dit et permet la saillance de l'image associée imposée ; mais elle peut aussi servir d'armure protectrice contre la force conservatrice des représentations doxiques.

La partie qui, dans le présent numéro, est consacrée aux figures de rhétorique, s'ouvre sur l'article de Marc Bonhomme, auteur de nombreux travaux de recherches sur les figures en général et sur la métonymie en particulier. Il y explore les lieux où " rhétoricité » et " argumentativité » figurales interfèrent et replace cette exploration dans le cadre de l'histoire de la rhétorique, de l'Antiquité à nos jours. Il propose une "version forte» de la thèse de l'argumentativité des figures, conçues comme «des formes condensées d'arguments " ou «concentrés de lieux » pouvant constituer des arguments à part entière et donc passer du domaine de l'élocution à celui de l'invention. Les prises de position épistémologiques sont complétées et enrichies par diverses illustrations qui accordent une place de choix au cas de la métonymie dans le discours publicitaire (signalons que le parallélisme structurel entre argument et figure ressort aussi, dans ce numéro, de la référence de Plantin à l'«argumentation métonymique »). Si Bonhomme pense avec Eggs et Snoeck Henkemans que la spécificité de l'argumentation figurale réside dans un pouvoir de dissimulation proche de celui du 
paralogisme, il envisage cependant la possibilité de ne pas l'interroger en termes de validité, mais en termes d'efficacité et de réussite de la visée argumentative.

Christian Plantin, éminent théoricien de l'argumentation en France, revisite l'histoire de l'opposition entre rhétorique des figures et rhétorique des arguments en problématisant la notion d'ornamentum, et propose une analyse critique du point de vue de la Nouvelle Rhétorique de Perelman sur la contribution systématique des figures à la construction des arguments. Cette analyse démontre que les auteurs du Traité de l'argumentation tentent de constituer dans le cadre de leur réflexion sur les figures une "sémantique du discours argumentatif " entendue comme une "théorie linguistique du discours ». Seule une telle sémantique serait en mesure de rendre compte du rôle rhétorique des concepts de "choix, présence et communion » (également évoqués par Snoeck Henkemans dans son étude de la mise en mots discursive de la prétérition). La théorie rhétorique des figures ne serait donc pas un "supplément fallacieux » de la "théorie classique du discours argumentatif», mais l'un de ses constituants intrinsèques.

Francisca Snoeck Henkemans, membre de la dynamique équipe d'Amsterdam sur la théorie de l'argumentation, présente un exemple de ses travaux actuels sur les figures de style dans le cadre de la pragma-dialectique. Elle problématise les pratiques discursives et les enjeux argumentatifs de la prétérition dans le cadre développé par van Eemeren et Houtlosser. La «tension potentielle» entre les visées «à la fois dialectique et rhétorique " de l'écriture y est présentée comme pouvant être résolue par des procédures d'« ajustement stratégique ». Ce mode de gestion de la discordance consiste, en l'occurrence, dans la concomitance de stratégies de dénégation de l'intention de dire, et d'énonciation contradictoire du contenu prétendument relégué dans le non-dit. La saillance de ce contenu est donc renforcée par sa propre dénégation. La coopération de l'auditoire est alors sollicitée d'autant plus fortement que l'énonciateur n'assumerait pas la responsabilité de ses dires et mettrait ainsi l'auditoire dans l'obligation d'assumer seul la prise en charge d'une interprétation évaluative dépréciative. L'argumentativité de la figure est donc explorée ici au prisme d'une prise de position normative.

Ekkehard Eggs, éminent spécialiste allemand de rhétorique classique, d'argumentation et de pragmatique et auteur (en français) d'une Grammaire du discours argumentatif. Le topique, le générique, le figuré (1994), revisite le cas exemplaire de l'ironie. La feintise inhérente à l'argumentativité figurale est à la fois confirmée et dépassée par un argumentaire qui attribue à l'ironie une stratégie de simulation constituant une fin en soi. L'enjeu véritable ne serait donc pas l'argumentation du «contrarium» entendu comme prise de position antithétique ou critique d'« un comportement, d'une opinion ou d'un lieu commun" parce qu' il pourrait et devrait en être autrement", mais l'invitation à considérer la mise en mots ironique comme l'indice saillant d'une rupture dans l'ordre des choses. La transparence de l'ironie résiderait donc paradoxalement dans l'affichage intentionnel de son opacité sémantique. On pourrait voir dans la saillance rhétorique de la "(dis)simulation transparente » conceptualisée par l'auteur un trait fondateur de l'« argumentation sui generis » de la figure.

Il me semble cependant que "(dis)simulation transparente" pourrait constituer le dénominateur commun de bien des figures; les articles de ce numéro le prouvent ainsi que ceux du numéro de Langue française 160 dont on lira ici-même le compte rendu. Cette formule percutante réfère en effet simultanément à l'opacité sémantique de la 
figure, à la feintise qui lui est inhérente et à la monstration intentionnelle de cette dernière... Que ne ferait-on afin de pouvoir « dire et ne pas dire » et de préserver sa liberté tout en rendant aux doxa l'honneur qui leur est dû? Que ne ferait-on afin de se libérer de l'obligation de rendre des comptes ou de justifier ses prises de position sans avoir à renoncer pour autant aux gratifications et aux plaisirs de la persuasion ou de la séduction?

\section{BIBLIOGRAPHIE}

Amossy, Ruth. 2006 [2000]. L'argumentation dans le discours (Paris : Colin)

Angenot, Marc. 1982. La parole pamphlétaire (Paris : Payot)

Anscombre, Jean-Claude \& Oswald Ducrot. 1988. L'Argumentation dans la langue (Liège : Mardaga)

Barthes, Roland. 1970. « L'ancienne rhétorique Aide-mémoire », Communications 16, Recherches

Rhétoriques, 172-223

Berrendonner, Alain. 2001-2002. « Portrait de l'énonciateur en faux naïf », Semen 15, 113-125

Bonhomme, Marc (éd.). 2001-2002. Figures du discours et ambiguité, Semen 15, 9-10

Bonhomme, Marc. 2001-2002. « De l'ambiguïté figurale », Semen 15, 11-24

Bonhomme, Marc. 2005. Pragmatique des figures du discours (Paris : Champion)

Blair, J. Anthony \& Ralph H Johnson. 1987. « Argumentation as Dialectical », Argumentation 1, 41-56

Breton, Philippe. 1996. L'argumentation dans la communication (Paris : La Découverte)

Carel, Marion \& Oswald Ducrot. 1999. « Le problème du paradoxe dans une sematique

argumentative », Langue française 123, 6-26

Charaudeau, Patrick. 2008. "L'argumentation dans une problématique d'influence ", Argumentation et Analyse du Discours, n 1, (http://aad.revues.org/index193.html. Consulté le 18 février 2009)

Douay-Soublin, Françoise. 1990. « Non, la rhétorique française au $18^{\mathrm{e}}$ siècle n'est pas "restreinte" aux tropes », Histoire Epistémologie Langage 12 :1, 123-132

Douay-Soublin, Françoise. 1990. « "Mettre dans le jour d'apercevoir ce qui est" : Tropologie et argumentation chez Dumarsais ", Figures et Conflits Rhétoriques, Meyer, Michel \& Alain Lempereur (éds) (Bruxelles : Editions de l'Université de Bruxelles), 83-102

Douay-Soublin, Françoise. 1994. « Les figures de rhétorique : actualité, reconstruction, remploi », Langue française 101, 13-25

Doury, Marianne. 1997. Le débat immobile (Paris : Kimé)

Doury, Marianne \& Sophie Moirand (éds). 2004. L'argumentation aujourd'hui (Paris : Presses de la Sorbonne nouvelle) 
Eggs, Ekkehard. 1994. Grammaire du discours argumentatif. Le topique, le générique, le figuré (Paris : Kimé)

Eemeren, Frans H. van, Rob Grootendorst, Francesca Snoek Hoekemans (eds). 1996. Fundamentals of Argumentation Theory (Mahwah, NJ \& London: Lawrence Erlbaum)

Eemeren, Frans H. van \& Peter Houtlosser (eds). 2002. Dialectic and Rhetoric. The Warp and Woof of ArgumentationAnalysis (Dordrecht : Kluwer Academic Publishers)

Genette, Gérard. 1972. « La rhétorique retreinte », Figures III (Paris : Seuil)

Gilbert, Michael. 1997. Coalescent Argumentation (Mahwah, NJ : Erlbaum)

Groupe 䭅. 1970. Rhétorique générale (Paris : Seuil)

Hamblin, Charles Leonard. 1970. Fallacies (London: Methuen)

Hansen, Hans V., Christopher W. Tindale , Athena V. Colman (eds). 1998. Argumentation \& Rhetoric [CD-ROM] (St. Catherines : Ontario Society for the Study of Argumentation)

Hermès, Cognition Communication Politique 15-16. 1995. « Argumentation et Rhétorique »

Johnson, Ralph H. 1998. « Argumentative space : Logical and Rhetorical Approaches », Hansen, Hans V., Christopher W. Tindale, Athena V. Colman (eds.). 1998. Argumentation and Rhetoric [CDROM]. (St. Catherines: Ontario Society for the Study of Argumentation)

Klinkenberg, Jean-Marie. 1990. «Rhétorique de l'argumentation et rhétorique des figures », Figures et Conflits Rhétoriques. Meyer, Michel \& Alain Lempereur (éds) (Bruxelles : Editions de l’Université de Bruxelles), 115-138

Klinkenberg, Jean-Marie. 1996. «Septième leçon Rhétorique de l'argumentation et rhétorique des figures : sœurs ou ennemies? ", Sept leçons de sémiotique et de rhétorique (Toronto : Éditions du GREF)

Krabbe, Erik C.W. 1998. "Who is afraid of Figures of Speech ?», « Argumentative space : Logical and Rhetorical Approaches ", Hansen, Hans V., Christopher W. Tindale, Athena V. Colman (eds). 1998. Argumentation and Rhetoric [CD-ROM] (St. Catherines : Ontario Society for the Study of Argumentation)

Landheer, Ronald. 1994. « Les figures de rhétorique et leur actualité en linguistique », « Présentation », Langue française 101, 3-12

Leff, Michael. 1999. «Rhetorical Prolepsis and the Dialectical Tier of Argumentation », Proceedings of the Fourth International Conference of the International Society for the Study of Argumentation (Amsterdam : Sic Sat), 510-513

Leff, Michael. 2002. « The relation between Dialectic and Rhetoric in a Classical and a Modern Perspective ", Dialectic and Rhetoric. The Warp and Woof of Argumentation Analysis (Dordrecht : Kluwer Academic Publishers), 53-64.

Leff, Michael. 2003. «Tradition and Agency in Humanistic Rhetoric ». Philosophy and Rhetoric, 36 : 2, 135-147

Leff, Michael. 2006. «Up from Theory : Or I fought the Topoi and the Topoi won », Rhetorical Society Quarterly 36: 2, 203-211

Le Guern, Michel. 1981. « Métaphore et argumentation », L'argumentation (Lyon : Presses Universitaires de Lyon), 65-74 
Lempereur, Alain. 1990. «Les restrictions des deux néo-rhétoriques », Figures et Conflits Rhétoriques. Meyer. Michel et Alain Lempereur (Bruxelles : Editions de l'Université de Bruxelles), 139-158

Meyer, Michel. 2004. La rhétorique (Paris : PUF)

Meyer, Michel. 2005. Qu'est-ce que l'argumentation? (Paris : Vrin)

Meyer, Michel. 2008 [1988]. De la problématologie (Paris : PUF)

Meyer, Michel. 2008. Principia Rhetorica (Paris : Fayard)

Molinié, Georges. 1992. Dictionnaire de rhétorique (Paris : Librairie Générale Française)

Molinié, Georges. 1994. "Problématique de la répétition », Langue française 101, « Les figures de rhétorique et leur actualité en linguistique », 102-11

Molinié, Georges. 1995. «Stylistique et tradition rhétorique », Hermès, Cognition Communication Politique. 15, 119-128

Molino, Jean. 1979. « Métaphores, modèles et analogies dans les sciences », Langages 54, La métaphore, Molino, Jean, Françoise Soublin, Joëlle Tamine (éds), pp. 83-102

Monte, Michèle. 2008. «Le jeu des points de vue dans l'oxymore : polémique ou reformulation? », Langue Française 160, 37-53

Perelman, Chaim \& Lucie Olbrechts-Tyteca. 1970 [1958]. Traité de l'argumentation. La nouvelle rhétorique (Bruxelles : Éditions de l'Université de Bruxelles)

Plantin, Christian. 1990. Essais sur l'argumentation (Paris : Kimé)

Plantin, Christian. 2005. L'argumentation (Paris : PUF)

Rabatel, Alain. 2008. "Introduction : figures et point de vue en confrontation », Langue Française 160 , « Figures et points de vue », 3-19

Rabatel, Alain. 2008. «Point de vue en confrontation dans les antimétaboles », Langue Française 160 , « Figures et points de vue », 21-36

Reboul, Olivier. 1986. «La figure et l'argument », De la métaphysique à la rhétorique, Meyer, Michel (éd.) (Bruxelles : Editions de l'Université de Bruxelles), 175-187

Reboul, Olivier. 1991. «Peut-il y avoir une argumentation non rhétorique ? ", Lempereur, Alain (éd.). L'argumentation, Colloque de Cerisy (Liège : Mardaga)

Reboul, Olivier. 1991a. Introduction à la rhétorique (Paris : PUF)

Rescher, Nicholas. 1998. "The role of rhetoric in rational Argumentation ", Hansen, Hans V., Christopher W. Tindale, Athena V. Colman (eds). Argumentation \& Rhetoric [CD-ROM] (St. Catherines: Ontario Society for the Study of Argumentation)

Tindale, Christopher W. 1998. "Introduction: The Importance of Rhetoric for Argumentation ", Hansen, Hans V., Christopher W. Tindale, Athena V. Colman (eds). Argumentation \& Rhetoric [CDROM] (St. Catherines: Ontario Society for the Study of Argumentation)

Tindale, Christopher W. 2004. Rhetorical Argumentation. Principles of Theory and Practice (London : Sage Publications)

Walton, Douglas. 1985. Arguer's Position : A Pragmatic Study of Ad Hominem Attack, Criticism, Refutation and Fallacy (Westport, Connecticut : Greenwood Press)

Walton, Douglas. 1992. The Place of Emotion in Argument (University Park, Pa.: Penn State Press) 
Walton, Douglas. 1997. Appeal to Pity: Argument ad Misericordiam (Albany: SUNY Press)

Walton, Douglas. 2000. Scare Tactics: Arguments that Appeal to Fear and Threats (Dordrecht: Kluwer Academic Publishers)

\section{NOTES}

1. «Un locuteur fait une argumentation lorsqu'il présente un énoncé E1 (ou un ensemble d'énoncés) comme destiné à en faire admettre un autre (ou ensemble d'autres) E2 » (Anscombre et Ducrot $1988: 8$ ).

2. Pierre de la Ramée (1515-1572), professeur au Collège royal à Paris.

3. C'est par l'opposition de ces deux "positions extrêmes" que s'ouvre l'ouvrage de Reboul: parlant de Perelman et Olbrechts-Tyteca, d'un côté, et de Morier, Genette, J. Cohen et le Groupe $\mu$, de l'autre côté, il note : « pour les premiers, la rhétorique vise à convaincre ; pour les seconds, elle constitue ce qui rend un texte littéraire » (1991:2-3).

4. Voir l'article «Informal Logic» dans le Stanford Encyclopedia of Philosophy, http:// plato.stanford.edu/entries/logic-informal.

5. «Ce qui caractérise la pratique de l'argumentation telle que la comprend la logique informelle est que son telos n'est pas de produire un consensus ou de parvenir à clore la discussion de quelque manière que ce soit, mais plutôt de réaliser un consensus dans lequel les parties acceptent que la force du meilleur raisonnement, et elle seule, a déterminé l'issue » (Johnson $1998: 3,2$. Je traduis).

6. Voir l'article de Tindale dans ce même numéro. Un premier palier est l'ensemble des prémisses qui mènent à une conclusion, un deuxième palier serait selon Johnson constitué par les objections et points de vue alternatifs auxquels l'argumentateur se doit d'apporter une réponse rationnelle.

7. Ainsi une liste de quinze numéros de revues publiés entre 1970 et 1984, recensés par Langue Française 101 : 11, mais aussi les publications mentionnées dans la note 1 de la « Présentation » de Semen 15, 2002 : 9 : «Poétique 36 (1978), Langage 54 (1979), Le Français moderne 51/4 (1983), Verbum 1, 2 et 3 (1993), Langue Française 101(1994) et 129 (2001). V. enfin Langue Française 160 (2008 : 3, n. 2 et 3). Hermès 15 et 16, "Argumentation et rhétorique ", accorde une place minime à la question des figures.

8. Cf., quant à l'histoire de la conception argumentative du rôle des figures, Bonhomme 2005 : 178 , ainsi que, pour ces dix à quinze dernières années, des commentaires comme celui de Douay (1994 : 14) qui souligne le fait que la réduction à une conception purement stylistique des figures commence à s'inverser, à l'époque de la rédaction de l'article, « au profit de l'argumentation ».

9. Cf., entre autres, à propos du caractère « inquiétant » de la division entre une « rhétorique des figures, propre à la littérature, et une rhétorique des conflits ", Meyer (2008 : 125), Molinié (1995 : 119).

10. Pour l'assimilation de la figure de style à un ornement présenté comme une fin en soi, v. Perelman et Olbrechts-Tyteca: "Nous considérerons une figure comme argumentative si, entraînant un changement de perspective, son emploi paraît normal par rapport à la nouvelle situation suggérée. Si, par contre, le discours n'entraîne pas l'adhésion de l'auditeur à cette forme argumentative, la figure sera perçue comme ornement, comme figure de style » (1970 : 229).

11. Angenot écrit à ce propos: "Nous ne prétendons pas traiter ces phénomènes [les figures] comme un ornatus qui se surajouterait au niveau argumentatif du discours. Ils influent sur l'intensité persuasive de celui-ci » (1982: 236). 
12. Bonhomme insiste toutefois dans son article « De l'ambiguïté figurale » sur le fait que si « le couplage Figuralité-Ambiguïté paraît s'imposer, il est loin d'être implicatif (2001-2002 : 11).

13. Cf., par exemple, à propos de la métaphore argumentative, Le Guern: «ce sont les métaphores argumentatives qui nous apportent les informations les plus solides sur le sémantisme de la langue. La métaphore poétique nous renseigne beaucoup moins sur la langue que sur l'idiolecte du poète » (1981: 72 - ainsi que 74).

14. Lire ici-même le compte rendu de ce numéro récent de Langue Française (160).

15. J'emprunte cette qualification à Tindale (2004: 74).

16. Tindale (2004: 71) emprunte la notion de « figural logic » à Fahnestock 1999. Ce qui renforce considérablement, dès le départ, la pertinence du point de vue de la rhétoricienne américaine, c'est le choix du discours scientifique comme principe d'organisation du corpus. Il est impossible de prétendre qu'un argumentaire scientifique recourt à des figures purement décoratives pour justifier ses thèses. Ses enjeux ne sont-ils pas inéluctablement marqués au sceau du savoir et de la logique? Cf., également, au sujet du rôle fondamental de la figuralité dans le discours des sciences exactes, Molino 1979.

17. Cf. Tindale 2004 (72-73) : " polyptoton : repeating a word in different grammatical cases », soit « electric », « electrified » et « electrised » dans la trame d'un discours scientifique.

18. Cf. Molinié (1992: 55) : «Une antimétabole est une figure microstructurale qui joue à la fois sur l'élocution et sur la construction. Elle consiste en effet en ce que, dans une phrase ou une suite de phrases, des groupes syntaxiques identiques se trouvent repris selon une permutation de leur dépendance interne. Ex. : Ce peuple paraît adorer le prince, et le prince adorer Dieu. Dans cet extrait de La Bruyère, l'antimétabole [...] réside dans l'inversion fonctionnelle adorer le prince - le prince adorer. Elle est toujours très marquée. "

19. L'ironie partage ce statut avec la métaphore dans la plupart des cas; nous évoquerons cependant le cas de cette figure dans la dernière partie de l'introduction, dans le cadre de l'analyse des concepts de saillance et de condensation.

20. Bonhomme (2005 : 184-185) considère l'ambiguité, le flou et la « duplicité » de l'ironie comme le second trait distinctif fondamental de l'argumentativité figurale. Sur la thèse de la duplicité et de la feintise, cf. également Bonhomme 2001-2002 (11-13, 15, 20, 22-24).

21. Cf., à ce sujet Reboul (1986:187) qui insiste sur le fait que la rhétorique argumentative étant nécessairement interactionnelle, un surcroît de force est nécessaire : «on dialogue avec tout son être. D'où la figure ".

22. Cf. le Traité de l'argumentation. La nouvelle rhétorique (1970 : 499-549) ; Reboul (1986: 182-3) ; Tindale (2004 : 65). V. également au sujet de la métonymie, " argument condensé », Reboul (1986 : 178) et Bonhomme (2005: 203-219).

23. Cf., au sujet des figures de l'assertion où la preuve et l'aplomb sont « confondus ", Angenot (1982: 238-244), mais aussi ce que Bonhomme (2005: 181) considère comme un des deux "facteurs illocutoires", spécifiques de l'argumentativité des figures : la capacité de "courtcircuitage ", soit la propension à imposer comme jugement de fait ce qui n'est que jugement de valeur et donc à « court-circuiter » les procédures de justification. 


\section{AUTEURS}

\section{RUTH AMOSSY}

Université de Tel Aviv, ADARR

ROSELYNE KOREN

Université Bar-Ilan, ADARR 